\title{
Peridynamic Modelling of Thermo-Oxidative Damage Evolution in a Composite Lamina
}

\author{
Selda Oterkus ${ }^{1}$ \\ The University of Strathclyde, Glasgow, UK \\ and \\ Erdogan Madenci ${ }^{2}$ \\ The University of Arizona, Tucson, AZ, 85721, USA
}

\begin{abstract}
Surface oxidation degrades the durability of polymer marix composites operating at high temperatures due to the presence of strong coupling between the thermal oxidation and structural damage evolution. The mechanism of oxidation in polymer matrix composites leads to shrinkage and damage growth. The thermo-oxidative behavior of composites introduces changes in diffusion behavior and mechanical response of the material. This study presents the derivation of peridynamic formulation for the thermo-oxidative behavior of the polymer matrix composites. As a demonstration purposes, isothermal aging of a unidirectional composite lamina is presented by using peridynamics. Oxidation contributed to the damage growth and its propagation.
\end{abstract}

\section{Introduction}

The thermo-oxidative environment results in growth of the thermo-oxidative layer and changes in material properties. The degradation mainly occurs on the surface layer, and it leads to weight loss and shrinkage of the oxidized layer. Therefore, prediction of long-term durability requires the consideration of both the oxidative changes and damage evolution.

The previous studies on the investigation of coupled thermo-chemo-mechanical models such as those of Zohdi [1], Wang [2], Gigliotti [3-4], Oskay [5] and Liang and Pochiraju [6] utilized finite element method (FEM) for the solution of the field equations in conjunction with a damage variable for monitoring material stiffness degradation. Zohdi [1] developed a FE model to address the coupling of thermo-chemo-mechanical processes in multiphase solids. The model is general enough to predict the loss of structural integrity of heterogeneous solids exposed to environmental attacks: diffusion, reactions, production of heat, changes in the stress field. Wang [2] considered a micromechanics FE model to develop high-temperature constitutive equations of polymer matrix composites with oxidation reaction, damage and degradation. Based on the irreversible thermodynamic theory, the model predicts oxygen concentration, mass loss and oxidation induced stresses.

Tandom et al.[7] used Galerkin FEM to compute the oxidative region growth and investigated the effect of fiber and interface diffusivity on the oxidation growth by using diffusion reaction model. Gigliotti [3-4] employed FEM to develop a unified approach based on the thermodynamics of irreversible processes. This study included not only the effect of the chemical field on the mechanical field, but also the effect of the mechanical field on the chemical field. Caglar [5] developed a computational multiscale and multiphysics approach by using FEM. Damage was monitored at each scale through an internal state variable. Recently, Liang and Pochiraju [6] developed a FE model for degradation of polymer matrix composites due to oxidation reactions and damage evolution. Their study explicitly modeled the time-dependent growth of oxidation layers, and utilized the eXtended FEM (XFEM).for evolution of discrete cracking in a homogenized representation of the composite. Liang et al. [8] considered the oxidation induced stress in G30/PMR-15 lamina with a pre-existing crack, and presented self-similar crack growth by using XFEM.

\footnotetext{
${ }^{1}$ Assistant Professor, Department of Naval Architecture Ocean and Marine Engineering, Member AIAA.

${ }^{2}$ Professor, Department of Aerospace and Mechanical Engineering, Associate Fellow AIAA
} 
These techniques suffer from the need to supply an external criterion for crack initiation and growth in the presence non self-similar and multiple crack paths. Considering the difficulty in obtaining and generalizing experimental fracture data, providing such a kinetic relation for crack growth clearly presents a major obstacle to fracture modeling with conventional methods, especially in a 3-D analysis. Even mesh free codes based on the conventional theories require these kinetic relations. The same is true of Cohesive Zone Elements (CZE) and eXtended Finite Element Method (XFEM) through injection of a local enrichment function.

The analysis of damage due thermal oxidation is fundamentally multi-physical and multi-scale in nature. Therefore, this study presents the PD modeling of isothermal aging of a unidirectional composite lamina. The oxidation degradation is modeled by coupling chemical and mechanical responses of the material and damage evolution. It incorporates multiple physical phenomena under one computational framework, peridynamics, which directly and fully couples the distinct physics involving mechanics of deformation, diffusion of temperature, oxygen concentration. The effects of material failure/damage is immediately influence local material properties governing the fields of diffusion. The PD theory enables the coupling of different fields [9-17].

\section{Deformation and damage prediction in a lamina}

The peridynamic (PD) theory introduced by Silling [18] is a non-local continuum approach, and it remains valid whether or not a discontinuity exists in the form of a crack. Its governing equation involving only integration rather than differentiation in space is given as

$$
\rho(\mathbf{x}) \ddot{\mathbf{u}}(\mathbf{x}, t)=\int_{H} f\left(\mathbf{x}^{\prime}-\mathbf{x}, \mathbf{u}^{\prime}-\mathbf{u}\right) d V^{\prime}+\mathbf{b}(\mathbf{x}, t)
$$

in which $f\left(\mathbf{x}^{\prime}-\mathbf{x}, \mathbf{u}^{\prime}-\mathbf{u}\right)$ represents the force density vector between material points $\mathbf{x}$ and $\mathbf{x}^{\prime}$, and it can be expressed as

$\mathbf{f}\left(\mathbf{u}^{\prime}-\mathbf{u}, \mathbf{x}^{\prime}-\mathbf{x}, t\right)=\mu \operatorname{css} \frac{\xi+\boldsymbol{\eta}}{|\xi+\boldsymbol{\eta}|}$

The force density vector is a function of initial relative position, $\boldsymbol{\xi}=\mathbf{x}^{\prime}-\mathbf{x}$ and relative displacement, $\boldsymbol{\eta}=\mathbf{u}^{\prime}-\mathbf{u}$ vectors. The parameter, $s$ represents the stretch between material points $\mathbf{x}^{\prime}$ and $\mathbf{x}$. The history-dependent scalarvalued function, $\mu$ facilitates the exclusion of the interactions when the interaction is removed in the form

$$
\mu\left(\mathbf{x}_{(j)}-\mathbf{x}_{(k)}, t\right)= \begin{cases}1 \text { if } s\left(\mathbf{x}^{\prime}-\mathbf{x}, t^{\prime}\right)<s_{c} & \text { for all } 0<t^{\prime}<t \\ 0 & \text { otherwise }\end{cases}
$$

The damage at a point is represented by the ratio of eliminated interactions to the total number of interactions of a material point as [19]

$$
\varphi(\mathbf{x}, t)=1-\frac{\int_{H} \mu\left(\mathbf{x}^{\prime}-\mathbf{x}, t\right) d V^{\prime}}{\int_{H} d V^{\prime}}
$$

In the case of a lamina, the PD material parameter $c$ has a directional dependency [20] in the form

$$
c=\left\{\begin{array}{cc}
c_{f}+c_{m} & \phi=\theta \\
c_{m} & \phi \neq \theta
\end{array}\right.
$$

where $c_{f}$ represents the PD material parameter in the fiber direction, and $c_{m}$ in the remaining directions. As shown in Fig. 1, the PD parameters are introduced for matrix and fiber with an orientation of $\theta$ from the horizontal 
axis. In this figure the material point $(p)$ interacts with the material point $(k)$ in the fiber orientation and the material point $(q)$ interacts with the material point $(k)$ in other orientations. The orientation of a PD interaction between the material point $(k)$ and the material point $(p)$ is defined by the angle $\phi$ with respect to the $x$-axis.

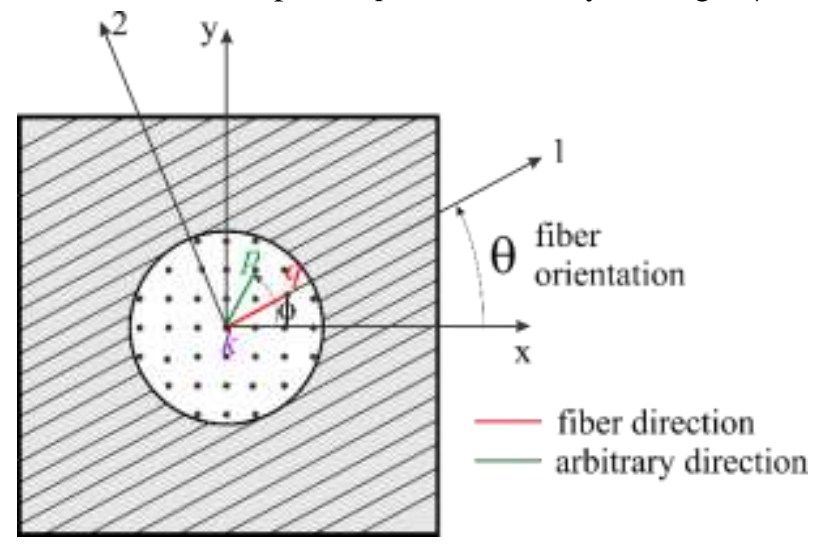

Figure 1. PD horizon for a lamina with a fiber orientation of $\theta$ and interaction of material point $(k)$ with the other material points within its horizon.

By considering simple loading conditions, Oterkus and Madenci [19] derived the PD material parameters in terms of the elastic modulus in the fiber direction, $E_{1}$, elastic modulus in the transverse direction, $E_{2}$, in-plane shear modulus, $G_{12}$, and in-plane Poisson's ratio, $v_{12}$ as

$c_{f}=\frac{2 E_{1}\left(E_{1}-E_{2}\right)}{\left(E_{1}-\frac{1}{9} E_{2}\right)\left(\sum_{q=1}^{Q} \xi_{q i} V_{q}\right)}$ and $c_{m}=\frac{8 E_{1} E_{2}}{\left(E_{1}-\frac{1}{9} E_{2}\right) \pi h \delta^{3}}$

with constraints on

$G_{12}=\frac{v_{12} E_{2}}{1-v_{21} v_{12}}=\frac{E_{1} E_{2}}{3\left(E_{1}-\frac{1}{9} E_{2}\right)}$ and $v_{12}=\frac{1}{3}$

These constraints can be removed by using ordinary state based PD formulation as described by Madenci and Oterkus [21].

\section{Therno-oxidation prediction in a lamina}

The surface of polymer composites exposed to oxidizing environment absorbs oxygen. The dissolved oxygen activates the oxidizing reaction inside the structure. The oxidation state variable, $\varphi$ indicates the extend of oxidation reaction [8].

PD diffusion-reaction equation with orthotropic oxygen diffusivity can be represented as

$\frac{\partial C}{\partial t}=\int_{H} \mu\left(\lambda \frac{C^{\prime}-C}{\left|\mathbf{x}^{\prime}-\mathbf{x}\right|}\right) d V^{\prime}-R_{c}(\varphi, \mathrm{T}, C)$

where $C$ is the oxygen concentration (molar volume), $R_{c}$ is the chemical reaction rate that is dependent on the oxidation state variable, $\varphi$ as

American Institute of Aeronautics and Astronautics 
$R_{c}(\varphi, \mathrm{T}, C)=\left\{\begin{array}{cc}R(\mathrm{~T}, C) & \text { for } \varphi>\varphi_{o x} \\ 0 & \text { for } \varphi=\varphi_{o x}\end{array}\right.$

in which $\varphi_{o x}$ represents the completely oxidized state.

The PD parameter for diffusion is represented by $\lambda$. Its directional dependency can be expressed as

$\lambda=\left\{\begin{array}{cc}D_{f}+D_{m} & \phi=\theta \\ D_{m} & \phi \neq \theta\end{array}\right.$

where $D_{f}$ and $D_{m}$ represent the PD diffusion parameters in the fiber and remaining directions [22]. These parameters can be derived in terms of the diffusion coefficients in the fiber and transverse directions, $D_{1}$ and $D_{2}$, respectively, as

$$
D_{f}=\frac{2\left(D_{1}-D_{2}\right)}{\sum_{q=1}^{N_{f}} \xi V_{q}} \text { and } D_{m}=\frac{6 D_{2}}{\pi \delta^{3} h}
$$

The diffusivity and the reaction rates depend on the aging temperature [8]. The solubility of oxygen defining the saturation molar volume absorbed at the surface also depends on the temperature. As shown in Fig.2, the oxidation state variable, $\varphi$ varies between its un-oxidized value of 1 and its completely oxidized value of $\varphi_{o x}$. It is related to the reaction rate, $R$ and completely oxidized state, $\varphi^{o x}$ as [8]

$$
\varphi=\max \left\{\varphi_{o x},\left(1-\int_{0}^{t} \gamma R(\zeta) d \zeta\right)\right\}
$$

with $\gamma$ represents the proportionality constant between weight loss and reaction rate.

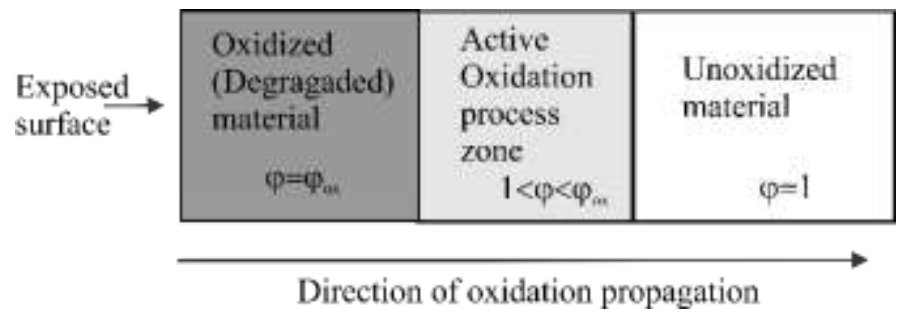

Figure. 2 Schematic view of thermo-oxidation zones

The temperature dependent reaction rate can be expressed as [7]

$R(C, T)=2 R_{o}(T) \frac{\beta C}{1+\beta C}\left(1-\frac{\beta C}{2(1+\beta C)}\right)$

with

$R_{o}(T)=R_{o}^{\prime} \exp \left(-\frac{R_{a}}{R_{u} T}\right)$ 
where $R_{o}^{\prime}$ and $\beta$ are the material specific reaction rate parameters, $R_{o}$ is the rate constant, $R_{a}$ is the activation parameter, $R_{u}$ is the universal gas constant and $T$ is the aging temperature.

The diffusion coefficients for the oxidized and un-oxidized materials are given in Arrhenius form [23] as

$$
\begin{aligned}
& D^{o x}=D_{0}^{o x} \exp \left(-\frac{E_{a}}{R_{u} T}\right) \\
& D^{u n}=D_{0}^{u n} \exp \left(-\frac{E_{a}}{R_{u} T}\right)
\end{aligned}
$$

where $E_{a}$ is the activation energy, and $D_{0}$ is a diffusivity constant. The oxidation dependent diffusion coefficient can be obtained as

$$
D(\varphi)=D^{u n}\left(\frac{\varphi-\varphi_{o x}}{1-\varphi_{o x}}\right)+D^{o x}\left(\frac{1-\varphi}{1-\varphi_{o x}}\right)
$$

Similarly, the oxidation induced stretch can be evaluated as;

$$
\Delta s=\alpha^{u n}\left(\frac{\varphi-\varphi_{o x}}{1-\varphi_{o x}}\right)+\alpha^{o x}\left(\frac{1-\varphi}{1-\varphi_{o x}}\right)
$$

where $\alpha$ is the shrinkage coefficient. The shrinkage coefficient of a lamina is dependent on the orientation of the material points $(k)$ and $(p)$ as

$$
\alpha(\phi)=\alpha_{x} \cos ^{2}(\phi)+\alpha_{y} \sin ^{2}(\phi)+\alpha_{x y} \sin (\phi) \cos (\phi)
$$

\section{Numerical results}

Thermo-oxidation in PMR-15 resin strip without a crack

The PD thermo-oxidation model is verified by considering a rectangular PMR-15 resin strip with length $L=200 \mu \mathrm{m}$ and width $W=20 \mu \mathrm{m}$. Along the left edge, oxygen absorption is allowed by specifying $C=C_{s}$ and oxidation absorption is not allowed on the remaining edges by specifying $\nabla C . \mathbf{n}=0$ with $\mathbf{n}$ representing unit normal vector to the boundary.

Table 1. Diffusivity parameters [7]

\begin{tabular}{ccc}
\hline & Unoxidized, $\varphi=1$ & Oxidized, $\varphi=\varphi_{o x}$ \\
\hline$D_{o}\left(\mathrm{~m}^{2} / \mathrm{s}\right)$ & $6.1 \times 10^{-11}$ & $8.9 \times 10^{-11}$ \\
\hline$E_{a}(\mathrm{~J} / \mathrm{mol})$ & 19700 & \\
\hline$R(\mathrm{~J} /(\mathrm{mol} \mathrm{K}))$ & 8.31447 & \\
\hline
\end{tabular}

Table 2. Reaction Rate Parameters for PMR-15 resin [7] 


\begin{tabular}{cc}
\hline Parameter & Value \\
\hline$R_{o}^{\prime}$ & $536.94 \mathrm{~mol} /\left(\mathrm{m}^{3} \mathrm{~min}.\right)$ \\
\hline$R_{a}$ & $23379.63 \mathrm{~J} / \mathrm{mol}$ \\
\hline$\beta$ & $0.92 \mathrm{~m}^{3} / \mathrm{mol}$ \\
\hline
\end{tabular}

The weight loss model parameters are specified as [7]

$\gamma=\left\{\begin{array}{cc}0.01-\frac{0.01-0.0033}{40} t, & t<40 \mathrm{~h} \\ 0.0033, & t>40 \mathrm{~h}\end{array}\right.$

$\varphi_{o x}=0.18$

Fig. 3 shows the variations of oxidation thickness at $288^{\circ} \mathrm{C}$. The oxidation layer increases as the oxygen concentration on the boundary increases. The results are similar to those obtained by Tandom et al. [23]. Fig. 4 shows the prediction of oxidation state variable for $C_{s}=2 \mathrm{~mol} / \mathrm{m}^{3}$. The color blue represents the oxidized (degragaded) region, the color red represents the un-oxidized region, and the other colors represent the active oxidation process zone. The size of the oxidized zone increases with the aging time.

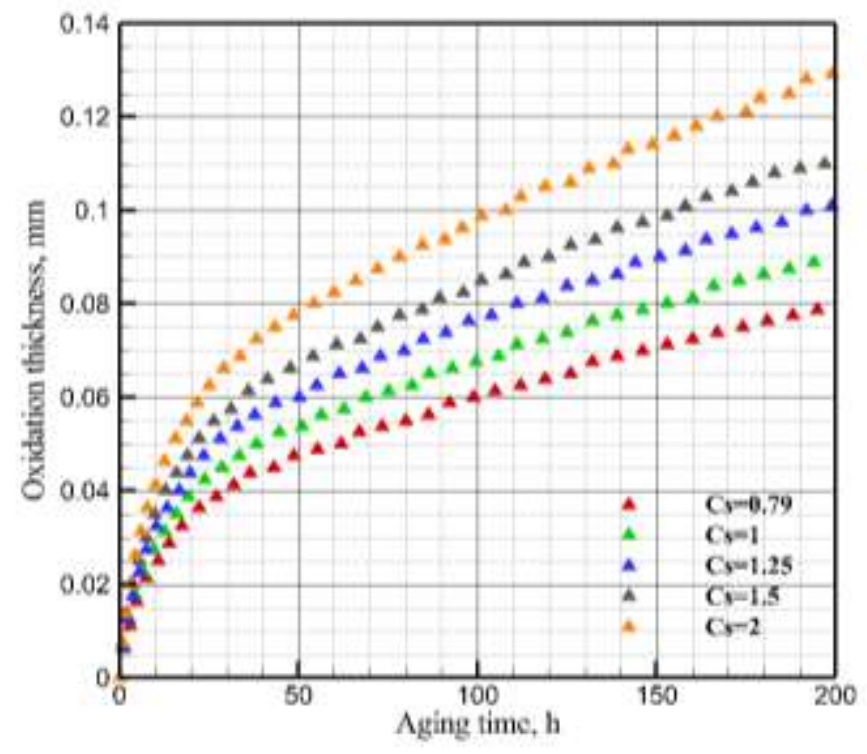

Figure 3. Oxidation layer growth prediction at $T=288^{\circ} \mathrm{C}$

\section{(a)}

(b)
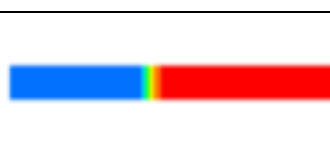

(a)
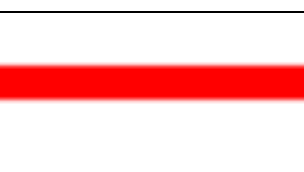


\section{Oxidation in G30/PMR-15 lamina with a crack}

The PD thermo-oxidation model is verified by considering a rectangular G30/PMR-15 lamina with a pre-existing crack. As shown in Fig. 5, it has length $L=200 \mu \mathrm{m}$ and width $W=20 \mu \mathrm{m}$ with a crack size $a=25 \mu \mathrm{m}$. Oxygen absorption is allowed on left edge as well as the crack surfaces by specifying $C=C_{s}$ and oxidation absorption is not allowed on the remaining edges by specifying $\nabla C . \mathbf{n}=0$ with $\mathbf{n}$ representing unit normal vector to the boundary. The critical energy release rate value of $G_{c}=0.14 \mathrm{~J} / \mathrm{m}^{2}$ is assumed to investigate the crack propagation of the oxidized lamina.

The pre-existing crack is located first symmetrically at $y=W / 2$, and subsequently non-symmetrically at $y=W / 4$. Fig. 6 shows the prediction of oxidation state variable for two crack configuration. The color blue represents the oxidized (degraded) region, the color red represents the un-oxidized region. The presence of crack increases the size of the oxidized region further. In both crack configurations, the size of the degraded region is about the same, but when the crack is at the center the oxidized region accumulates at the center. The final crack size in both cases is about $26 \mu m$.

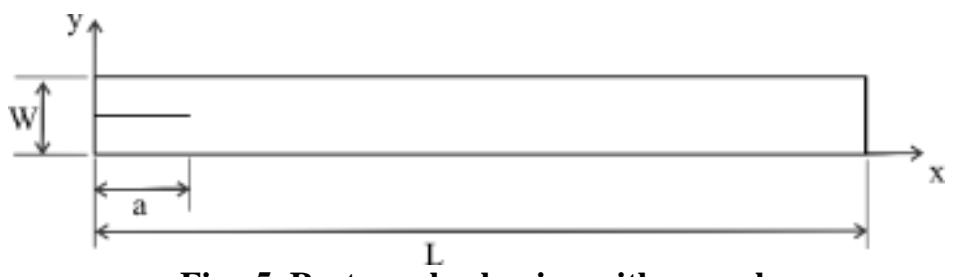

Fig . 5 Rectangular lamina with a crack

Table 3. Material properties for G30/PMR-15 lamina

\begin{tabular}{lcccc}
\hline & $\mathrm{E}_{1}(\mathrm{GPa})$ & $\mathrm{E}_{2}(\mathrm{GPa})$ & $\mathrm{E}_{2}(\mathrm{GPa})$ & $\mathrm{G}_{12}(\mathrm{GPa})$ \\
\hline Unoxidized & 120.9 & 7.64 & 0.313 & 3.81 \\
\hline Oxidized & 121.4 & 8.74 & 0.313 & 4.59 \\
\hline
\end{tabular}

Table 4. Material properties for G30/PMR-15 lamina

\begin{tabular}{lll}
\hline Diffusivity & $\mathrm{D}_{1}\left(\mathrm{~mm}^{2} / \mathrm{h}\right)$ & $\mathrm{D}_{2}\left(\mathrm{~mm}^{2} / \mathrm{h}\right)$ \\
\hline Unoxidized & $6.75 \mathrm{E}-03$ & $0.8 \mathrm{E}-03$ \\
\hline Oxidized & $9.83 \mathrm{E}-03$ & $1.17 \mathrm{E}-03$ \\
\hline
\end{tabular}

Table 5. Material properties for G30/PMR-15 lamina

\begin{tabular}{lll}
\hline Shrinkage coefficients & $\alpha_{1}(1 / \mathrm{h})$ & $\alpha_{2}(1 / \mathrm{h})$ \\
\hline Unoxidized & $-4.80 \mathrm{E}-08$ & $-1.89 \mathrm{E}-06$ \\
\hline Oxidized & $-2.60 \mathrm{E}-07$ & $-8.29 \mathrm{E}-06$ \\
\hline
\end{tabular}




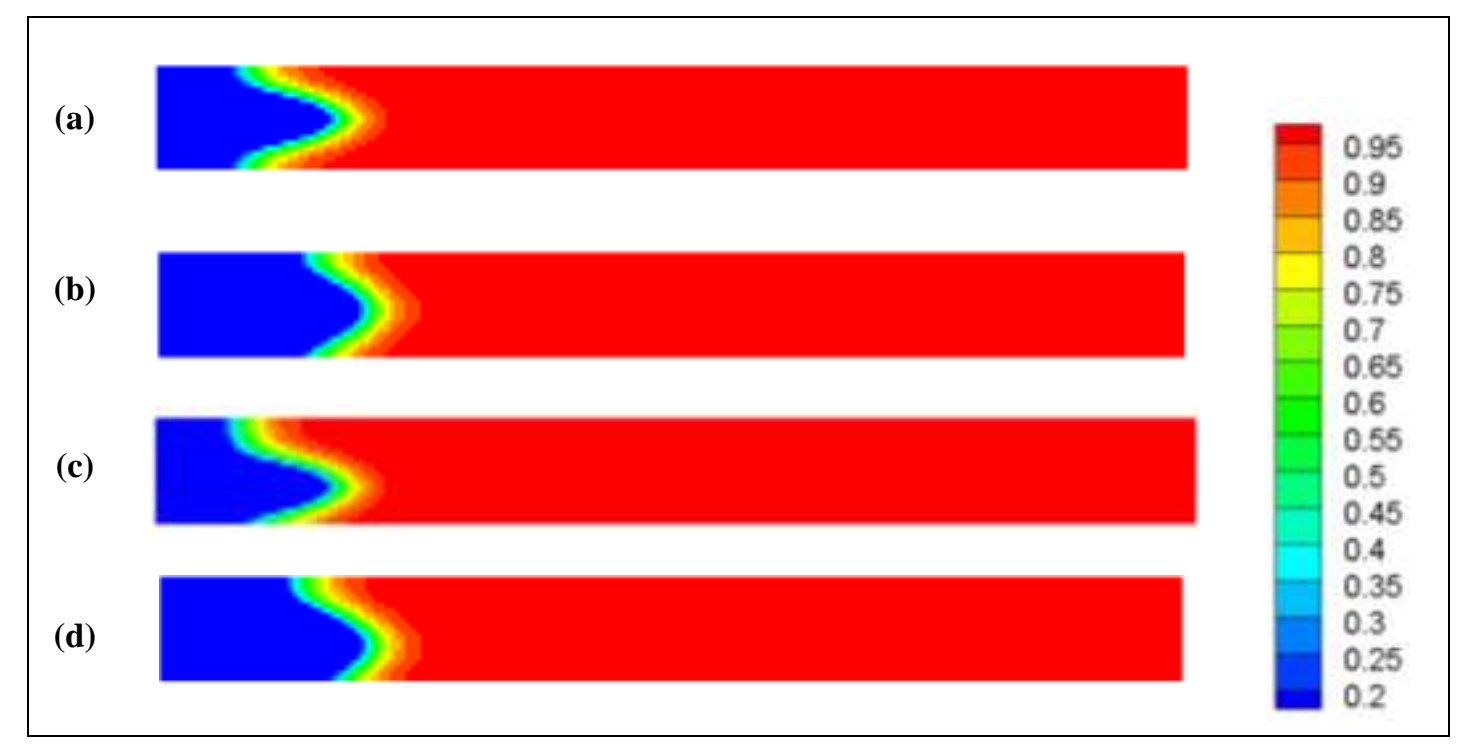

Figure 6 Variation of oxidation state variable at $T=288^{\circ} \mathrm{C}$ for $C_{s}=0.79$
a) $t=2.5 \mathrm{~h}($ crack at $y=W / 2)$, b) $t=3 \mathrm{~h}($ crack at $y=W / 2)$
c) $t=2.5 \mathrm{~h}($ crack at $y=W / 4)$, d) $t=3 \mathrm{~h}($ crack at $y=W / 4)$

\section{Remarks}

This study presents the development of peridynamic formulation for thermo-oxidative behavior of polymer matrix composites. The diffusion reaction model and wight loss effects are included in the peridynamic formulation.The peridynamic modeling thermo-oxidative response of a composite lamina is successfully simulated. The effect of damage growth on the thermo-oxidation and the oxidation layer growth are simulated. The oxidation lead to cracking in the composite material. The peridynamic results agree well with the previous studies.

\section{References}

[1] Zohdi, T. I., Modeling and simulation of a class of coupled thermo-chemo-mechanical processes in multiphase solids. Comput. Methods Appl. Mech. Engrg, 2004, Vol. 193, pp. 679-699.

[2] Wang, S. S. and Chen, X., Computational Micromechanics for High-Temperature Constitutive Equations of Polymer-Matrix Composites with Oxidation Reaction, Damage, and Degradation, Journal of Engineering Materials and Technology, 2006, Vol. 128, pp. 81-89

[3] Marco Gigliotti, M. and Grandidier, J.-C., Chemo-mechanics couplings in polymer matrix materials exposed to thermooxidative environments, 2010, C. R. Mecanique, Vol. 338, pp. 164-175

[4] Gigliotti, M, Grandidier, J.-C. and Lafarie-Frenot, M. C., Assessment of chemo-mechanical couplings in polymer matrix materials exposed to thermo-oxidative environments at high temperatures and under tensile loadings, 2011, Mechanics of Materials, Vol. 43, pp. 431-443

[5]Oskay,C., and Haney, M. Computational modeling of titanium structures subjected to thermo-chemo-mechanical environment, International Journal of Solids and Structures, 2010, Vol. 47, pp. 3341-3351

[6] Liang, J. and Pochiraju, K. V., Oxidation-induced damage evolution in a unidirectional polymer matrix composite, Journal of Composite Materials, 2015, Vol. 49, pp.1393-1406

[7] Tandon, G. P., K. V. Pochiraju, and G. A. Schoeppner. "Thermo-oxidative behavior of high-temperature PMR-15 resin and composites." Materials Science and Engineering: A 498.1 (2008): 150-161.

[8] Liang, Jianyong, and Kishore V. Pochiraju. "A methodology for the estimation of transverse failure strength of an oxidized lamina from isothermal aging studies." Composites Part A: Applied Science and Manufacturing 73 (2015): 101-108. 
[9] Oterkus S, Madenci E, Agwai A., Fully Coupled Peridynamic Thermomechanics. Journal of the Mechanics and Physics of Solids, 2014, Vol. 64, pp. 1-23.

[10] Oterkus S., Madenci E, Oterkus E, Hwang Y, Bae J, Han S., Hygro-Thermo-Mechanical Analysis and Failure Prediction in Electronic Packages by Using Peridynamics, 2014, 64th Electronic Components \& Technology Conference, Lake Buena Vista, FL, USA.

[11] Oterkus, S., \& Madenci, E. (2013). Crack growth prediction in fully-coupled thermal and deformation fields using peridynamic theory. 54th AIAA Structures, Structural Dynamics and MaterialsConference, Boston, Massachusetts.

[12] Oterkus, S. (2015). Peridynamics for the solution of multiphysics problems.

[13] Oterkus, S., Fox, J., \& Madenci, E. (2013, May). Simulation of electro-migration through peridynamics. In 2013 IEEE 63rd Electronic Components and Technology Conference (pp. 1488-1493). IEEE.

[14] Han, S., Lim, S., Bae, J., Hwang, Y., Lee, S., Oterkus, S., Madenci, E., Diyaroglu, C. and Oterkus, E., 2015 , May. Equivalent acceleration assessment of JEDEC moisture sensitivity levels using peridynamics. In 2015 IEEE 65th Electronic Components and Technology Conference (ECTC) (pp. 1518-1523). IEEE.

[15] Chen, Z., \& Bobaru, F. (2015). Peridynamic modeling of pitting corrosion damage. Journal of the Mechanics and Physics of Solids, 78, 352-381.

[16] De Meo, D., Diyaroglu, C., Zhu, N., Oterkus, E., \& Siddiq, M. A. (2016). Modelling of stress-corrosion cracking by using peridynamics. International Journal of Hydrogen Energy, 41(15), 6593-6609.

[17] Madenci, E., \& Oterkus, S. (Eds.). (2016). Chapter 16 Peridynamics for Coupled Field Equations. In Handbook of Peridynamic Modeling (pp. 489-530). CRC Press.

[18] Silling, S. A., 2000, "Reformulation of Elasticity Theory for Discontinuities and Long-Range Forces," Journal of Mechanics and Physics of Solids, Vol. 48, pp. 175-209.

[19] Silling, S.A., Askari, E., (2005), A meshfree method based on the peridynamic model of solid mechanics. Comput. Struct. 83, 526-1535.

[20] Oterkus, Erkan, and Erdogan Madenci. "Peridynamic analysis of fiber-reinforced composite materials." Journal of Mechanics of Materials and Structures 7.1 (2012): 45-84.

[21] Madenci, E. and Oterkus. E., 2014, Peridynamic Theory and Its Applications, Springer, Boston, MA.

[22] Oterkus, Selda, and Madenci, Erdogan "Fully coupled thermomechanical analysis of fiber reinforced composites using peridynamics." 55th AIAA/ASME/ASCE/AHS/SC Structures, Structural Dynamics, and Materials Conference-SciTech Forum and Exposition 2014.

[23] Tandon, G. P., K. V. Pochiraju, and G. A. Schoeppner. "Modeling of oxidative development in PMR-15 resin." Polymer Degradation and Stability 91.8 (2006): 1861-1869. 